\title{
Rejets radioactifs des centrales nucléaires et environnement $\left(^{*}\right)$
}

\author{
J.-J. MARTIN (**) \\ (Manuscrit reçu le 17 septembre 1975)
}

\begin{abstract}
RÉSUMÉ
Après avoir dressé le bilan des rejets radioactifs gazeux et liquides des centrales nucléaires en service ou en projet, on donne une méthode simple d'appréciation de la dose pour les voies directes d'irradiation de l'homme que constituent l'inhalation et la consommation d'eau de boisson. Les résultats complets de l'estimation portant sur toutes les voies d'irradiation directes ou indirectes font apparaître que la dose annuelle extrême est de quelques millirems par an pour un site tel que Fessenheim. Les contrôles dans l'environnement, effectués tant par l'exploitant nucléaire que par la Santé Publique doivent permettre de confirmer ces estimations.
\end{abstract}

\begin{abstract}
Gaseous and liquid radioactive effluents from operating or scheduled nuclear power plants are reviewed and a simple method of dose assessment for direct pathways of human exposure resulting from inhalation and tap water is given. The complete results of the assessment of all the direct or indirect exposure pathways show that the highest annual dose is to be a few millirems per year for a site such as Fessenheim. Environmental controls carried out both by the nuclear operator or Public Health Services should verify these assessments.
\end{abstract}

Cet exposé nous permettra, tout d'abord, de dresser le bilan de la radioactivité rejetée avec les effluents gazeux et liquides par les centrales nucléaires en service ou en projet en France. Nous examinerons, ensuite, l'incidence de ces rejets sur la dose subie par la population voisine et nous terminerons par une description rapide des contrôles effectués dans l'environnement.

(*) Communication présentée aux Journées d'Information sur l'Environnement nucléaire organisées à Paris du 4 au 6 février 1975 par l'Association pour le développement des Sciences et Techniques de l'Environnement.

$\left({ }^{* *}\right)$ Électricité de France, Service de la Production thermique, Département de Radioprotection, B.P. $\mathrm{n}^{\circ} 120,93203$ Saint-Denis. 


\section{INTRODUCTION}

Une première difficulté apparaît lorsque l'on évoque, auprès du public, l'activité rejetée par les centrales nucléaires. Celle-ci est exprimée en curies (Ci) grandeur physique qui caractérise à la fois la masse de matière radioactive mise en jeu et son aptitude à se désintégrer.

Malheureusement, cette grandeur physique ne permet pas d'aboutir simplement à l'effet biologique sur l'individu. Des activités exprimées par la même valeur numérique en curies de différents nucléides peuvent avoir des effets sur l'homme excessivement différents.

Pour passer de l'activité rejetée à la dose subie par l'homme, il faut connaître les conditions de propagation ou de concentration dans le milieu naturel, l'utilisation de ce milieu par l'homme, et le devenir des radioéléments lorsqu'ils ont été introduits dans l'organisme.

Une première approche de ce problème est de considérer les concentrations maximales admissibles fixées, en ce qui concerne les matières radioactives, par la Commission Internationale de Protection Radiologique. Ces concentrations, exprimées en curies par mètre cube dans l'air ou dans l'eau, représentent la contamination du milieu qui, entretenue en permanence, conduirait à la dose maximale admissible chezl'homme. Encore ce dernier terme doit-il être bien compris comme une dose admissible et non comme une dose produisant des dégâts irréversibles. C'est un peu l'équivalent, que les puristes pardonnent cette approximation utilisée pour les besoins de la compréhension, du cachet d'aspirine que l'on pourrait absorber chaque jour et le rapport entre dose maximale admissible et dose léthale est aussi grand que le rapport entre l'unique cachet d'aspirine et la boîte complète.

A titre de comparaison, disons que la dose maximale admissible pour les personnes du public est sensiblement égale à cinq fois la dose due à l'irradiation naturelle.

Connaissant la concentration maximale admissible (CMA) très variable avec chaque radioélément, il est possible de convertir la valeur numérique de l'activité rejetée en une grandeur qui permettra la comparaison entre les doses résultant des rejets de différents nucléides, à savoir le volume d'air ou d'eau nécessaire pour diluer l'effluent jusqu'à obtenir la concentration maximale admissible. Cette méthode permet même la comparaison avec d'autres polluants que les produits radioactifs.

\section{BILAN DES REJETS}

Plutôt que de donner les résultats d'exploitation de centrales particulières qui sont fluctuants d'une année à l'autre pour un réacteur donné et variables en fonction des sites et de la puissance installée, il a paru préférable d'indiquer :

- les rejets extrêmes observés en fonction de l'énergie produite dans les réacteurs en service $(\mathrm{Ci} / \mathrm{TWh})$; 
- la valeur, exprimée dans la même unité, de l'activité prévue pour les deux tranches de la centrale de Fessenheim (1800 MW électriques);

- l'activité correspondante exprimée en curie par an;

- le volume d'air ou d'eau de dilution nécessaire pour diluer cette activité jusqu'à obtenir la concentration maximale admissible.

Ces grandeurs sont exprimées pour chacune des catégories d'effluents (gaz rares, aérosols et produits volatils pour les effluents gazeux; tritium et éléments autres que le tritium pour les effluents liquides) à l'intérieur desquelles la concentration maximale admissible des différents nucléides est relativement peu différente.

Le tableau I fait apparaître ces différents résultats.

TABLEAU I

ACTIVITÉS REJETÉES

Effluents liquides

- tritium

- autres que tritium

Effluents gazeux

- gaz nobles

- aérosols et produits volatils

\begin{tabular}{|c} 
Centrales en service \\
$\mathrm{Ci} / \mathrm{TWh}(1)$ \\
$100 / 1000(3)$ \\
$0,2 / 20$ \\
$000 / 15000$ \\
$0,01 / 3$
\end{tabular}

\begin{tabular}{|l|c|c|}
\multicolumn{3}{|c|}{ Fessenheim } \\
Ci/TWh & Ci/an & $\mathrm{m}^{3} / \mathrm{an}(2)$ \\
& & \\
250 & 2500 & $10^{6}$ \\
1,5 & 15 & $10^{7}$ \\
& & \\
3000 & 30000 & $3.10^{11}$ \\
0,15 & 1,5 & $7,5.10^{9}$
\end{tabular}

(1) Valeur minimale/valeur maximale.

(2) Volume de dilution nécessaire pour obtenir la concentration maximale admissible.

(3) BWR exclus.

S'agissant des effluents liquides, il est possible de juger des possibilités de dilution de ces effluents en considérant que le débit du canal d'Alsace est de l'ordre de $2.10^{10} \mathrm{~m}^{3} / \mathrm{an}$, et le seul débit de l'eau de circulation, qui assure le refroidissement des condenseurs de $10^{9} \mathrm{~m}^{3} /$ an. Ceci permet de conclure, en première approximation, que la contamination moyenne dans l'eau de circulation sera $1 \mathrm{p}$. mille de la CMA pour le tritium et $1 \mathrm{p}$. cent de la CMA pour les autres nucléides que le tritium. Si le public consommait l'eau de circulation en tant qu'eau de boisson, la dose annuelle représenterait le même rapport avec la dose maximale admissible. La dilution supplémentaire d'un facteur 20 apportée par le débit du canal d'Alsace réduit la dose dans la même proportion.

Concernant les effluents gazeux, il faut savoir qu'une cheminée basse, non particulièrement dégagée, comme le sont toutes celles des centrales nucléaires, permet un volume de dilution annuel, à $500 \mathrm{~m} \mathrm{du}$ point de rejet,

VOL. $10-\mathrm{N}^{\circ} 4$ 
de l'ordre de $10^{13} \mathrm{~m}^{3} /$ an (le volume de dilution augmente évidemment avec la distance; plus on s'éloigne du rejet, plus le panache s'étend en largeur). Donc, en première approximation, la concentration moyenne des gaz rares sera de l'ordre de $3 \mathrm{p}$. cent de la CMA à $500 \mathrm{~m}$, soit $3 \mathrm{p}$. cent de la dose maximale admissible pour une personne qui resterait en permanence à la clôture du site sous les vents dominants.

Cette méthode permet de comparer les rejets gazeux d'une centrale nucléaire avec ceux d'une centrale thermique. Une centrale au charbon (charbon moyen américain) de 1800 MWe rejette environ 270000 t/an de $\mathrm{SO}_{2}$ et $0,03 \mathrm{Ci} /$ an de radium 226 , élément radioactif naturel contenu dans le charbon. Pour obtenir la CMA de ces gaz, il faut un volume de dilution annuel de $3.10^{14} \mathrm{~m}^{3}$ pour le $\mathrm{SO}_{2}$ et de $3.10^{10} \mathrm{~m}^{3}$ pour le radium, c'est-à-dire dans le premier cas, un volume très supérieur à celui nécessaire pour une centrale nucléaire que l'on obtient en utilisant des cheminées élevées (débit de dilution disponible de $10^{15}$ à $10^{16} \mathrm{~m}^{3} / \mathrm{an}$ ).

\section{EXPOSITION DU PUBLIC}

La méthode approchée que nous venons d'utiliser permet d'avoir une idée des doses subies par les voies directes d'exposition que sont l'inhalation de l'air ou la contamination de l'eau de boisson.

Il existe d'autres voies d'irradiation du public, plus indirectes et plus complexes telles que la consommation des légumes ou du lait provenant

TABLEAU II

DOSE ANNUELLE ( $\mu \mathrm{rem} / \mathrm{an}$ )

Rejets gazeux Rejetsliquides Total

Fessenheim

- Valeur moyenne

- Valeur extrême

180

200

Chooz 1973

400

Rayonnement naturel

Dose maximale admissible
220

2100

350
400

2300

750

100000

500000

d'herbages contaminés par les effluents gazeux ou par l'irrigation avec l'eau de la rivière, la consommation de poissons, la baignade, le bain de soleil sur les plages des rivières ou des mers...

Le calcul des doses par l'intermédiaire de ces voies d'irradiation fait intervenir des études sur le transfert des radioéléments dans le milieu naturel 
et vivant et sur l'utilisation du milieu par l'homme. Il prend en compte, en particulier, les facteurs de concentration quelquefois très importants que l'on rencontre particulièrement chez les êtres vivants élémentaires.

Un tel calcul est actuellement appliqué pour tous les sites nucléaires. Quelques résultats figurent au tableau II où ils sont comparés au rayonnement naturel et à la dose maximale admissible pour les personnes du public. Celle-ci est prise égale à $500 \mathrm{mrem} / \mathrm{an}$, valeur admise pour les membres du public, car il s'agit ici de personnes du voisinage qui accumulent les doses par toutes les voies d'irradiation conjuguées : l'individu considéré habite l'habitation la plus exposée vis-à-vis des effluents gazeux, consomme le lait et les poissons les plus contaminés du voisinage de la centrale, etc. Ces résultats ne doivent pas être comparés à la dose génétique maximale admissible pour la population dans son ensemble (170 mrem/an); le calcul de cette dose génétique, pour autant qu'il soit possible de le conduire avec une approximation suffisante, donnerait vraisemblablement une dose inférieure de plusieurs ordres de grandeur.

Les calculs font donc apparaître que, pour les personnes du voisinage immédiat, la dose ajoutée au rayonnement naturel représente quelques centièmes de celle-ci et de l'ordre de un pour cent de la dose maximale admissible.

$\mathrm{Si}$ on admet la proportionalité de l'effet et de la dose, les effets éventuels de cette dose supplémentaire ne représenteront donc que quelques pour cent du rayonnement naturel, toutes voies d'irradiation confondues.

\section{CONTRÔLES DANS L'ENVIRONNEMENT}

Les contrôles commencent d'abord à la source, c'est-à-dire avant le rejet des effluents dans l'atmosphère ou dans le milieu naturel. Ils portent sur l'activité des gaz rares, des aérosols et des produits volatils (chaque catégorie nécessitant un processus de prélèvement et de mesure différent) et sur des prélèvements des effluents liquides (sur lesquels on analyse l'activité des différents nucléides : tritium, strontium 90 , émetteurs $\alpha$, émetteurs $\beta$ ). Ces contrôles permettent de dresser un bilan détaillé de l'activité rejetée de chaque radioélément.

Ces contrôles sont essentiels car eux seuls donnent la possibilité d'évaluer les doses résultant des rejets; en effet, la contamination dans l'environnement est si faible qu'elle est, soit non décelable, soit, grâce à des méthodes et moyens de mesure perfectionnés, mesurable avec une précision très relative.

Toutes les doses supportées par la population du fait des rejets des centrales nucléaires doivent donc être calculées, à défaut de pouvoir être mesurées dans la nature, encore moins sur l'individu.

Des contrôles systématiques sont, néanmoins, assurés périodiquement sur l'air (aérosols et eau de pluie), l'eau de rivière, la chaîne alimentaire (lait, légumes, poisson) ainsi que sur des indicateurs qui ne rentrent pas directement dans les voies d'irradiation de l'homme, mais sont des accumulateurs d'activité intéressants pour suivre une évolution (sédiments des fleuves ou 
des côtes, par exemple). Ces mesures permettent de s'assurer que les différentes voies d'irradiation de l'homme sont contrôlées et de confirmer ainsi les évaluations de dose.

Toutes ces mesures sont contrôlées par le Service Central de Protection contre les Rayonnements Ionisants du Ministère de la Santé qui reçoit, chaque mois, l'état des rejets de l'installation ainsi que des échantillons d'effluents pour analyse dans son propre laboratoire, systématiquement ou sur sa demande particulière. Ce Service effectue également des mesures déjà assurées par E.D.F. dans l'environnement, avec des moyens de mesure plus sensibles et dans le cadre d'un réseau de mesures qui lui permet d'effectuer des comparaisons entre les sites nucléaires et des stations de référence éloignées de toute activité nucléaire.

\section{CONCLUSION}

Le bilan des rejets des centrales nucléaires et les calculs de doses qu'il permet de faire, en tenant compte de toutes les voies possibles d'irradiation de l'homme, montrent que la dose résultant du fonctionnement de ces centrales n'est qu'une part réduite de la dose déjà subie par la population du fait des rayonnements naturels.

Les contrôles effectués sur les effluents et sur l'environnement, tant par l'exploitant nucléaire que par les services officiels de Santé Publique, nous paraissent présenter un caractère exceptionnel de sévérité qui est propre à l'industrie nucléaire et qu'il ne semble pas que l'on rencontre, à un degré aussi élevé, dans les autres branches industrielles. Ils peuvent constituer, à notre avis, la garantie que les rejets des centrales nucléaires sont, et resteront sans conséquences sanitaires appréciables pour la population. 